\title{
A novel topoisomerase I inhibitor DIA-001 induces DNA damage mediated cell cycle arrest and apoptosis in cancer cell
}

\author{
Jiaqi Liu ${ }^{1}$, Guohe Geng ${ }^{2}$, Guang Liang ${ }^{2}$, Ling Wang ${ }^{3}$, Kuntian Luo ${ }^{4,5}$, Jian Yuan ${ }^{4,5}$, Shiguang Zhao ${ }^{1}$ \\ ${ }^{1}$ Department of Neurosurgery, The First Affiliated Hospital of Harbin Medical University, Harbin 150001, China; ${ }^{2}$ School of Pharmaceutical \\ Sciences, Wenzhou Medical University, Wenzhou 325035, China; ${ }^{3}$ Department of Pharmacy, Fujian Provincial Hospital, Provincial Clinical College \\ of Fujian Medical University, Fuzhou 350001, China; ${ }^{4}$ Research Center for Translational Medicine, East Hospital, Tongji University School of \\ Medicine, Shanghai 200120, China; Key Laboratory of Arrhythmias of the Ministry of Education of China, East Hospital, Tongji University School \\ of Medicine, Shanghai 200120, China \\ Contributions: (I) Conception and design: J Yuan, K Luo, J Liu, G Liang and G Geng; (II) Administrative support: S Zhao; (III) Provision of study \\ materials or patients: J Yuan; (IV) Collection and assembly of data: J Liu; (V) Data analysis and interpretation: J Liu, L Wang; (VI) Manuscript \\ writing: All authors; (VII) Final approval of manuscript: All authors. \\ Correspondence to: Shiguang Zhao. Department of Neurosurgery, The First Affiliated Hospital of Harbin Medical University, Harbin 150001, \\ China. Email: guangsz@hotmail.com; Jian Yuan. Research Center for Translational Medicine, East Hospital, Tongji University School of Medicine, \\ Shanghai 200120, China. Email: yuanjian229@hotmail.com; Kuntian Luo. Research Center for Translational Medicine, East Hospital, Tongji \\ University School of Medicine, Shanghai 200120, China. Email: amethystian@hotmail.com.
}

Background: DNA topoisomerase enzyme plays an essential role in controlling the DNA topology
structure by binding to DNA and cutting the phosphate backbone of either one or both of the DNA strands.
Here, we have identified a small molecule inhibitor, DIA-001, that directly binds to Topoisomerase 1 (Topo I)
and promotes the Topo I-DNA adducts.

Methods: In this study, we investigated the antitumor effects of DIA-001 using MTS assay and colony formation. We examined cell cycle of tumor cells with DIA-001 treatment in vitro by flow cytometry. And we investigated DNA damage and cell cycle marker protein after treatment with DIA-001 at different concentration and time point by western blot. Immunofluorescence was performance to detect the nuclear foci. The effects of DIA-001 on Topo I and Topo II activities were examined by DNA relaxation assays.

Results: We demonstrate that DIA-001 inhibit DNA replication and arrest cell cycle progression at the G2/M phase by directly binds to Topo I and promotes the Topo I-DNA adducts. In addition, DIA-001 can activate the DNA damage response signaling cascade, resulting in apoptosis in treated cells.

Conclusions: Our findings show a novel compound for treatment of cancer cells with the potential as a chemotherapy candidate that is less toxic to normal cells.

Keywords: Topoisomerase I; cell cycle arrest; apoptosis; chemotherapy

Submitted Oct 21, 2019. Accepted for publication Dec 17, 2019.

doi: $10.21037 /$ atm.2019.12.138

View this article at: http://dx.doi.org/10.21037/atm.2019.12.138

\section{Introduction}

Topoisomerase I (Topo I) is a type IB enzyme with an essential role in controlling the topological structure of DNA during replication, transcription, recombination and repair (1-3). Topo I transiently cut one strand of DNA to form single-strand breaks, allowing supercoiled DNA to relax. For rapid cell division, cancer cells need high Topo
I activity to finish the DNA metabolic processes. Due to the specific function of Topo I, inhibiting Topo I can induce DNA double-strand breaks and cellular lethality (4). Therefore, Topo I inhibitors have been developed for cancer therapy (4). However, many Topo I inhibitors, such as camptothecin (CPT) are limited by poor solubility and dose limiting bone marrow toxicity, diarrhea, nausea, and 


\section{Page 2 of 8}

vomiting. The discovery of novel catalytic inhibitor for Topo I is needed.

Topo1 inhibitors are grouped as two types, (I) Topo1 poisons that covalently trap topoisomerases on DNA $(5,6)$. (II) Topo1 catalytic inhibitors, which prevent DNA cleavage $(5,6)$. In our study, the $3 Z)-3-[2-(4-$ Chlorophenyl)-2-oxoethylidene]-1,3-dihydro-2H-indol2-one $\left(\mathrm{C}_{16} \mathrm{H}_{10} \mathrm{ClNO}_{2}\right.$, DIA-001) has been discovered as a novel Topo1 poison and catalytic inhibitor. DIA-001can bind Topo1 to form a Topo1-drug complex that prevents the DNA replication process, induces cell cycle arrest at the G2/M phase and activates the apoptosis pathway. Here, we report the DIA-001 as a novel catalytic Topo1 inhibitor, which binds with the enzyme to decrease the efficiency of supercoiled DNA relaxation and induce DNA damage.

\section{Methods}

\section{Cell culture and antibodies}

The breast epithelial cell line MCF-10A, mouse muscle myoblast cell line C2C12, human glioma cell lines U251, T98G, LN18, human ovarian cancer cell line OVCAR8, human osteosarcoma cell lines U2OS, human hepatocellular carcinoma cell line HepG2 and human malignant melanoma cell line A375 were purchased from American Type Culture Collection (ATCC, USA). The identities of all these cell lines were confirmed by the medical genome facility at the Mayo Clinic Center (Rochester, MN, USA) using short tandem repeat profiling. Cells were maintained in the appropriate media with $10 \%$ FBS.

Antibodies against Chk1, phosphor-Chk1 (Ser345), Chk2, phosphor-Chk2 (Thr68), and cleavage PARP were purchased from Cell Signaling Inc. Anti- $\gamma \mathrm{H} 2 \mathrm{AX}$ (05-636) was purchased from Millipore. Anti- $\beta$-actin antibodies were purchased from Sigma. Antibodies against Cyclin A, Cyclin B and horseradish peroxidase-conjugated secondary antibodies against mouse (sc-2748) and rabbit (sc-2750) were purchased from Santa Cruz.

\section{MTS and clonogenic assay}

For MTS assay, the cells were plated on 96-well plates at densities of 2000 cells per well for U251, OVC8, U2OS, A375, LN18, HepG2 and T98G cell lines respectively in $100 \mu \mathrm{L} /$ well medium and incubated for 24 hours. Then, fresh medium was added and cells were treated with DIA001(TimTec) at different concentrations for 72 hours.

\section{Liu et al. A novel top I inhibitor induces DNA damage in cancer cell}

Cell viability was examined with MTS according to the manufacturer's instructions (Promega). Briefly, $20 \mu \mathrm{L}$ of MTS were added to each well 3 hours later. The absorbance at $490 \mathrm{~nm}$ was measured with a microplate reader (Tecan Infinite M1000 PRO).

For clonogenic assay, 500 cells were seeded in a 6-well plate for 24 hours and then treated with the indicated concentration of DIA-001. After 10 days, the cells were washed with PBS, fixed with methanol and stained using $0.1 \%$ crystal violet and the colony numbers were counted.

\section{Western blotting}

Cells were lysed with $0.5 \%$ NP40, 50 mMTris, $150 \mathrm{mM} \mathrm{NaCl}$, and $1 \mathrm{mM}$ EDTA (NETN) buffer with $10 \mathrm{mM} \mathrm{NaF}, 50 \mathrm{mM} \beta$-glycerophosphate, and $1 \mathrm{mg} / \mathrm{mL}$ each of aprotinin and pepstatin A. Proteins were separated by SDS-PAGE, transferred onto PVDF membrane and probed using appropriate primary and secondary antibodies. Finally, proteins were detected using ECL Western Blotting Detection Reagents (ThermoFisher).

\section{Immunofluorescence for nuclear foci}

Cells were seeded on a coverslips. After treatment, cells were washed with PBS and fixed with $3 \%$ paraformaldehyde for 15 minutes, permeabilized with $0.5 \%$ Triton- $\mathrm{X}$ in PBS for 5 minutes and following with blocking by $5 \%$ goat serum for 1 hour at room temperature. Then, cells were incubated with primary antibody at $4{ }^{\circ} \mathrm{C}$ overnight, followed by incubation with Alexa Fluor 594 or Alexa Fluor 488 conjugated secondary antibodies at $37{ }^{\circ} \mathrm{C}$ for 20 minutes. After washed with PBS, cell nuclei were counterstained with DAPI. The signals were examined by confocal microscopy.

\section{Cell cycle analysis}

Cells were collected and fixed with $70 \%$ ethanol at $-20{ }^{\circ} \mathrm{C}$ overnight, then washed with PBS twice. Cells were stained with propidium iodide (PI) containing RNase for 30 minutes in the dark, then analyzed for cell-cycle profile by FACS (Beckman Coulter). Data were analyzed using ModFit LT software.

\section{TOPO I and II mediated DNA relaxation assays}

The effects of DIA-001 on Topo I and Topo II activities were examined by the conversion of supercoiled pBR322 
DNA to its relaxed form using topoisomerase I and II drug screening kits (Topogen) according to the manufacturer's protocol. Briefly, The Topo I activity was studied in a $20 \mu \mathrm{L}$ reaction system including $1 \mu \mathrm{L}$ human Topo I, variable volume of DIA-001 (final concentrations: 10, $20 \mu \mathrm{M})$ or CPT $(50 \mu \mathrm{M}), 4 \mu \mathrm{L} 5 \times$ complete assay buffer, $1 \mu \mathrm{L}$ pBR322 DNA, and variable volume $\mathrm{H} 2 \mathrm{O}$ (to a final volume of $20 \mu \mathrm{L}$ ). These mixtures were incubated at $37^{\circ} \mathrm{C}$ for $30 \mathrm{~min}$ and the reaction stopped using $2 \mu \mathrm{L} 10 \%$ SDS. The samples were run on a $1 \%$ agarose gel with $0.5 \mu \mathrm{g} / \mathrm{mL}$ ethidium bromide at $50 \mathrm{~V}$. The gel was destained in water before being photographed under UV-light. The known Top I poisons CPT was used as the positive control. The effect on Topo II activity by DIA-001 was performed using a similar procedure of Topo I. The known Topo II inhibitor etoposide was used as the positive control.

\section{Statistics}

All the data are presented as mean $\pm \mathrm{SD}$. Statistical analysis was performed with Graphpad8 software (GraphPad Inc. USA). Two tailed Student's $t$-test was used to evaluate statistical significance between groups. $\mathrm{P}<0.05$ was considered significant difference. In the figures, the statistical significance is showed by: n.s. no significance, ${ }^{*} \mathrm{P}$ $<0.05 ;{ }^{* *} \mathrm{P}<0.01,{ }^{* * *} \mathrm{P}<0.001$.

\section{Results}

\section{DIA-001 inbibits the proliferation of cancer cells}

To evaluate the effect of DIA-001 on proliferation inhibition on different cancer cells, the MTS assay and colony formation assay were performed. The cytotoxicity was determined by IC50 calculations for treatment of U251, OVC8, U2OS, A375, LN18, HepG2 and T98G cells by DIA-001 were 1.987, 3.782, 2.425, 0.5399, 3.031, 8.279 and $14.20 \mu \mathrm{M}$ respectively (Figure $1 A, B, C, D, E, F, G, H$ ). Colony formation results showed the same growth inhibition on different cancer cell lines of DIA-001, while this compound did not have cytotoxic effect for normal cells MCF-10A and C2C12 in concentrations up to $1 \mu \mathrm{M}$ (Figure 1I, $7, K, L, M, N, O, P$ ). Collectively, these results indicate that DIA-001 has anti-tumor effect for different cancer cell lines.

\section{DIA-001 Induces DNA damage}

DNA damage is a common reason for inhibition of proliferation and cytotoxicity $(7,8)$. We investigated whether DIA-001 would cause DNA damage in U2OS cell through by examine the phosphorylation of the checkpoint kinase 1, 2 (p-Chk1/2), and $\mathrm{H} 2 \mathrm{AX}(\gamma \mathrm{H} 2 \mathrm{AX}) \cdot \gamma \mathrm{H} 2 \mathrm{AX}$ staining, a marker of DNA damage, was used to assess the level of DNA damage (9-11). As shown in Figure 2A,B,C, DIA-001 treatment induced $\gamma \mathrm{H} 2 \mathrm{AX}$ foci formation and the number of $\gamma \mathrm{H} 2 \mathrm{AX}$ foci increased in a time and dosedependent manner. Furthermore, western blot analysis showed that the DIA-001 treatment induced H2AX and Chk1/2 phosphorylation levels (Figure 2D). Collectively, these results indicate that DIA-001 markedly induced DNA damage in cancer cell.

\section{DIA-001 induces G2/M arrest and apoptosis in cancer cells}

The DNA damage checkpoint is a mechanism that triggers cell cycle arrest to allow enough time for repair (12-14). To uncover the mechanisms of DIA-001 in inhibiting cell proliferation, we studied the cell cycle profiles of U2OS cells after DIA-001 treatment. The result shows that after 24-hour of treatment with 10 and $20 \mu \mathrm{M}$ DIA-001, U2OS cells arrested at the G2/M phase (Figure $3 A, B$ ). Western blot analysis of the cell cycle regulators cyclin A and $\mathrm{B}$ in U2OS cell treated with 10 and $20 \mu \mathrm{M}$ DIA-001 for 24 hours also showed that DIA-001 induced an accumulation of cyclin A and a slight increase of cyclin B, supporting the G2/M arrest (15-17). We also examined whether DIA-001 could induce apoptosis. Cleavage of PARP serves as a marker for cells undergoing apoptosis (18-20). We found that PARP cleavage increased in U2OS cell at 24-hour of DIA-001 treatment (Figure 3C). Our results suggest that DIA-001 induces cell cycle arrest and apoptosis in cancer cell lines.

\section{DIA-001 causes accumulation of Topo1cc}

We next explored how DIA-001 causes DNA damage. Interestingly, we found that Topoisomerase might be a target. As shown in Figure 4A, we found the accumulation of Topo1 cleavage complexes (Top1cc) in U2OS cells 4 hours after DIA-001 treatment (CPT treatment was used as a positive control). We hypothesized that DIA-001 might be a Top1 inhibitor. To determine the mechanism of DIA001 induced Top1cc accumulation, we used the in vitro enzyme assays to test for topoisomerase activity. As shown in Figure 4B, supercoiled pHOT1 DNA cannot be relaxed by topo I with DIA-001 at 10 and $20 \mu \mathrm{M}$ (lane 5 and 6). To 
A
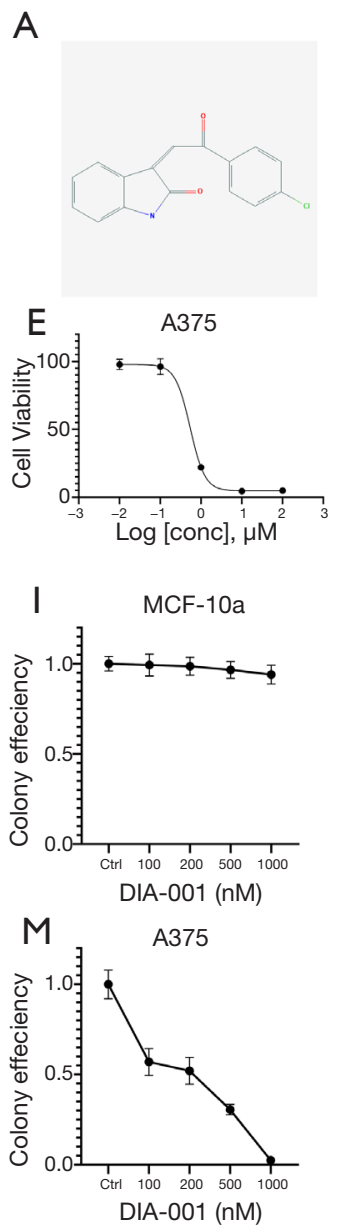

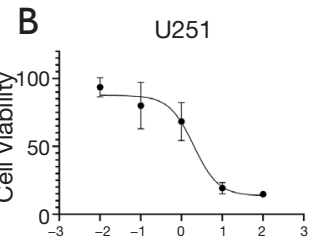

$\log [$ conc], $\mu \mathrm{M}$
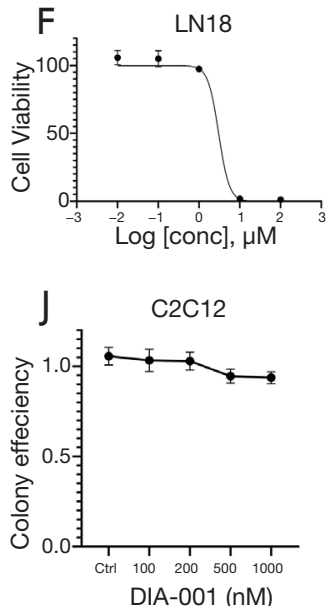

N

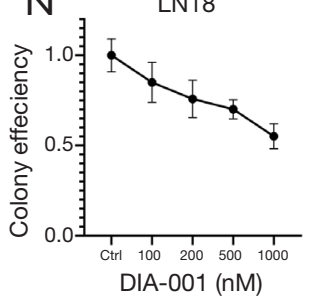

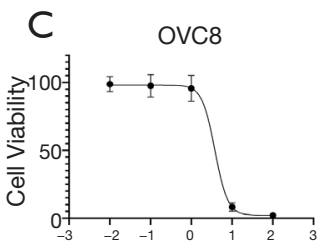

Log [conc], $\mu \mathrm{M}$

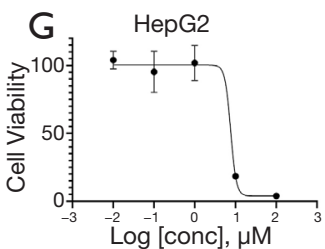

K OVC8

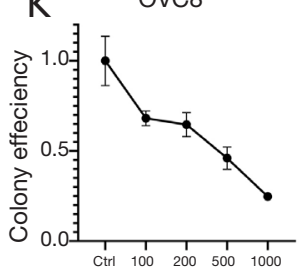

DIA-001 (nM)

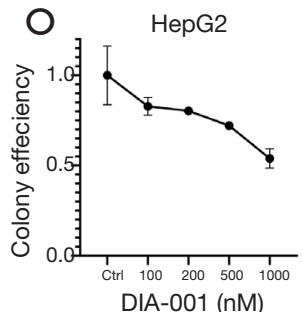

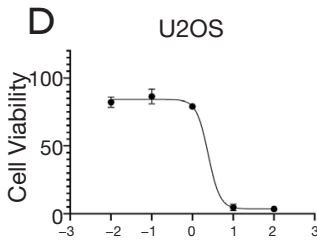

$\log [$ conc], $\mu \mathrm{M}$

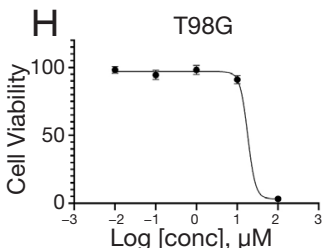

L U2OS

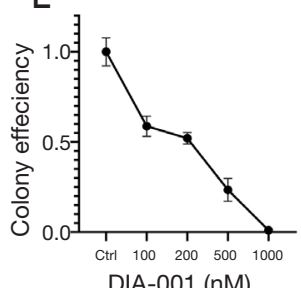

P T98G

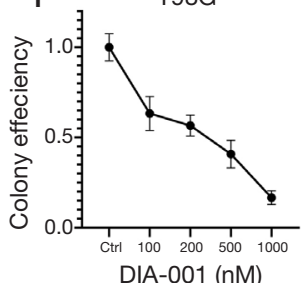

DIA-001 (nM)

Q

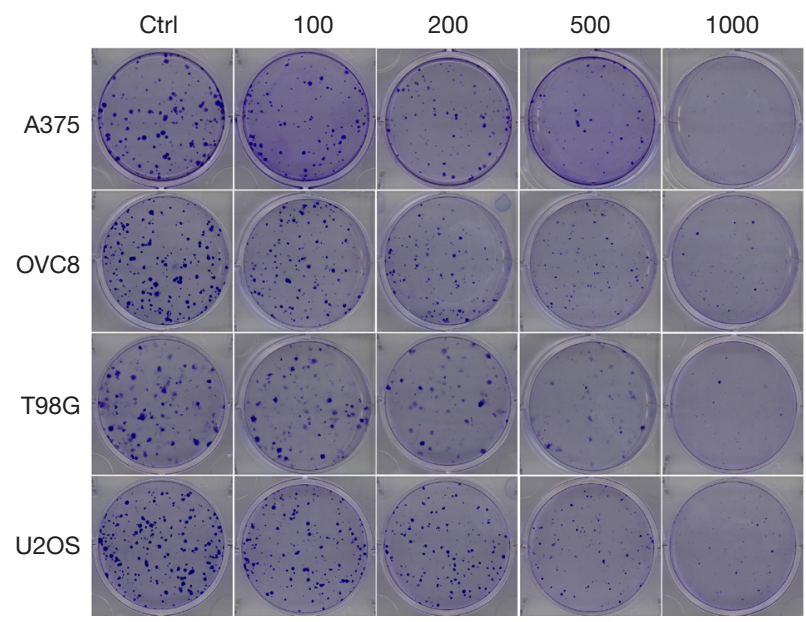

Figure 1 The cytotoxicity of DIA-001 on different cell lines. (A) The chemical structure of DIA-001. (B,C,D,E,F,G,H) DIA-001 treatment of different cancer cell lines. Cells were seeded into 96-well plates at 2,000 cells per well and were then treated with DIA-001 at various concentrations for 72 hours. Cell viability was assessed with MTS assay. (I,J,K,L,M,N,O,P) Clonogenicity of different normal and cancer cell lines upon DIA-001 treatment. 300 cells were seeded in triplicate in 6-well plates and treated with various concentrations of DIA-001 for 10 days, and colonies were counted (mean $\pm \mathrm{SD}$ ). (Q) Representative data show the colonies. 
A

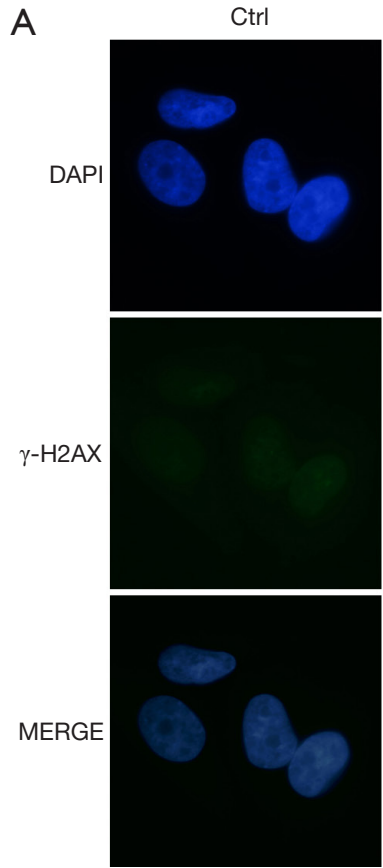

IR, 2Gy
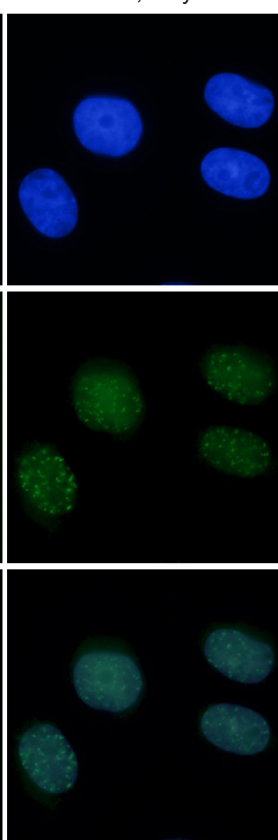

DIA-001

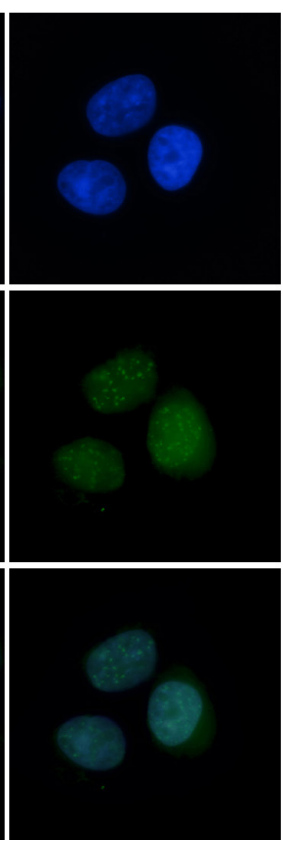

$B$
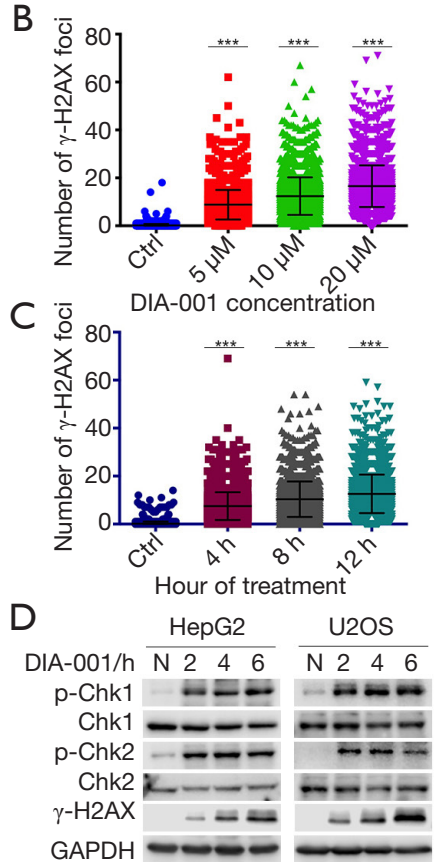

Figure 2 DIA-001 induces DNA damage. (A) $\gamma$-H2AX staining in U2OS cells treated with $20 \mu M$ of DIA-001, IR, 2Gy as positive control. (B) DIA-001 induced DNA damage is dose-dependent. (C) DIA-001 induced DNA damage is time-dependent. (D) Western blot analysis of $\gamma$-H2AX, Chk1, p-Chk1, Chk2 and p-Chk2 after indicated treatment. GAPDH was used as a loading control.

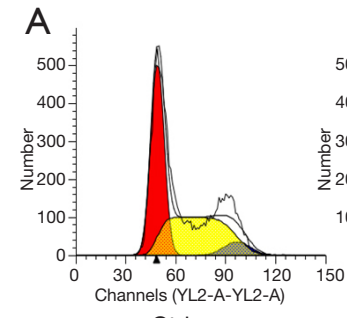

Ctrl

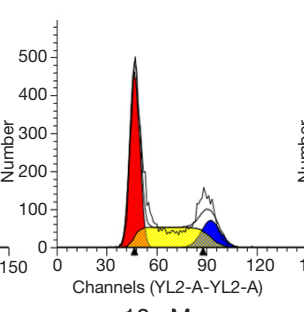

$10 \mu \mathrm{M}$

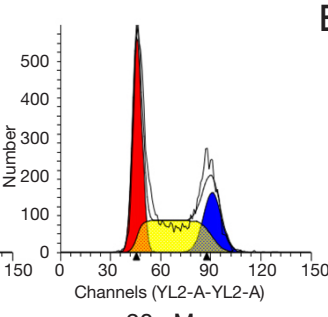

$20 \mu \mathrm{M}$
B

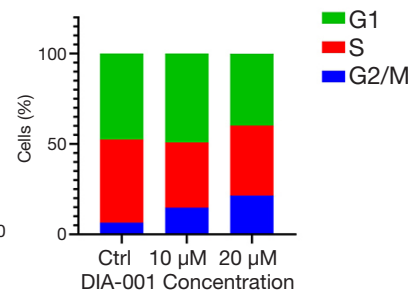

C

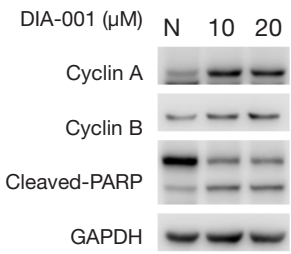

Figure 3 DIA-001 induces the cell cycle arrest at the G2/M phase. (A,B) The U2OS cells were treated with DMSO, 10 and $20 \mu M$ of DIA001 for 24 hours, followed by FACS analysis. (C) Western blot analysis of Cyclin A, Cyclin B and cleavage PARP after indicated treatment. GAPDH was used as a loading control. DMSO, dimethyl sulfoxide.

investigate whether the cytotoxicity of DIA-001 is specific for topo I, we used the kDNA decatentation assay, which measures topo II activity in vitro. The kinetoplast DNA $(\mathrm{kDNA})$ is an ideal substrate for topoisomerase II assays because it is specific for the TopoII reaction mechanisms. As shown in Figure $4 C$, kDNA was decatentated in the presence of topo II (lane 4) and in the presence of DIA001 (lane 6-7). In contrast, the topo II inhibitor, etoposide inhibited the decantenation (lane 5). To further elucidate the mechanism of DIA-001 inhibition through formation of a drug-enzyme-DNA complex, we analyzed the predicted topoisomerase enzyme binding site of DIA-001. As shown in Figure 4D,E, the 3-D structure of the Topo I and II shows DIA-001 fits into the binding site of Topo I rather than the Topo II.

\section{Discussion}

DNA topoisomerases were recognized as promising targets in cancer, considering its pivotal role in essential biological process (4,21-23). Currently approved topoisomerase inhibition therapies are directed toward blocking the 


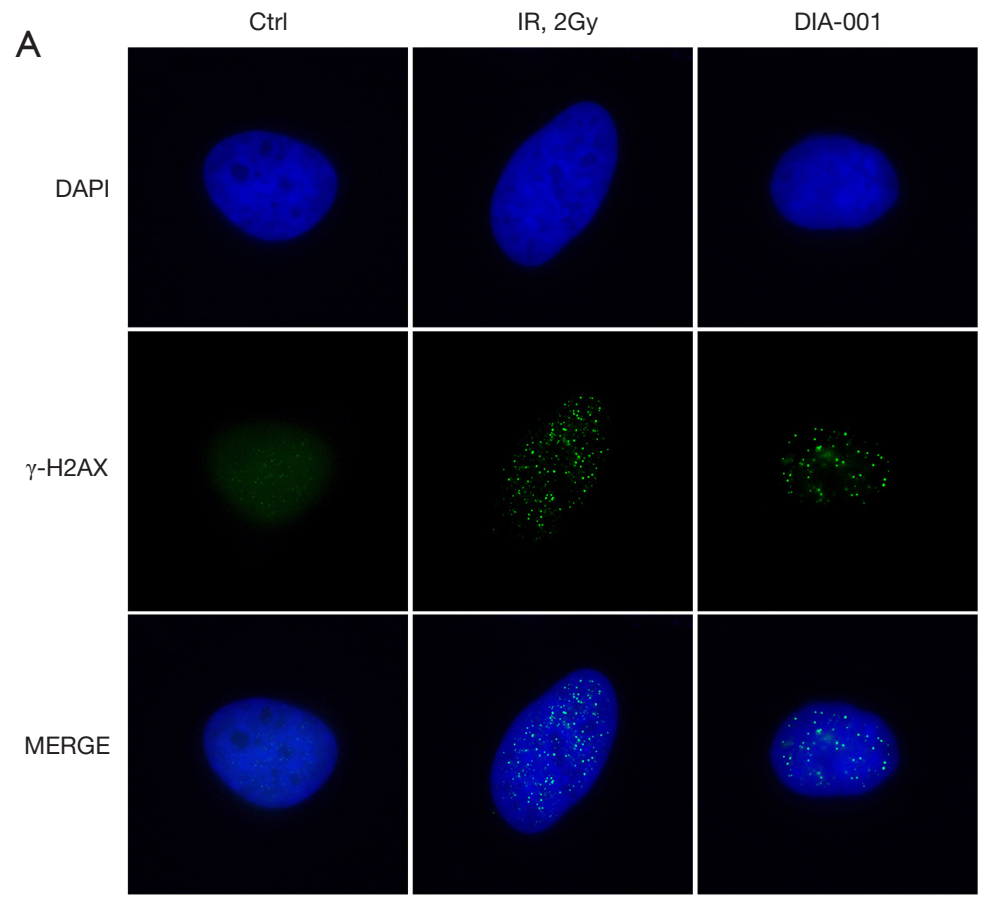

B

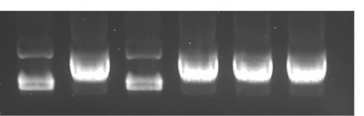

1. Relaxed pHOT-1 DNA maker

2. Supercoiled pHOT-1 DNA

3. Topo I + Supercoiled pHOT-1 DNA

4. Topo I + Supercoiled pHOT-1 DNA + CPT $(50 \mu \mathrm{M})$

5. Topo I + Supercoiled pHOT-1 DNA + DIA-001 $(10 \mu \mathrm{M})$

6. Topo I + Supercoiled pHOT-1 DNA + DIA-001 $(20 \mu \mathrm{M})$

C

E

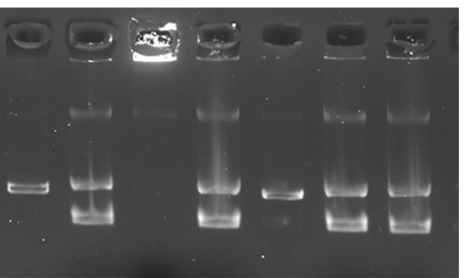

1. Linearized kDNA 2. Decatenated kDNA

3. Catenated kDNA 4. Topo II Catenated kDNA

5. Topo II Catenated kDNA + Etoposide $(100 \mu \mathrm{M})$

6. Topo II Catenated kDNA + DIA-001 $(10 \mu \mathrm{M})$

7. Topo II Catenated kDNA + DIA-001 $(20 \mu \mathrm{M})$
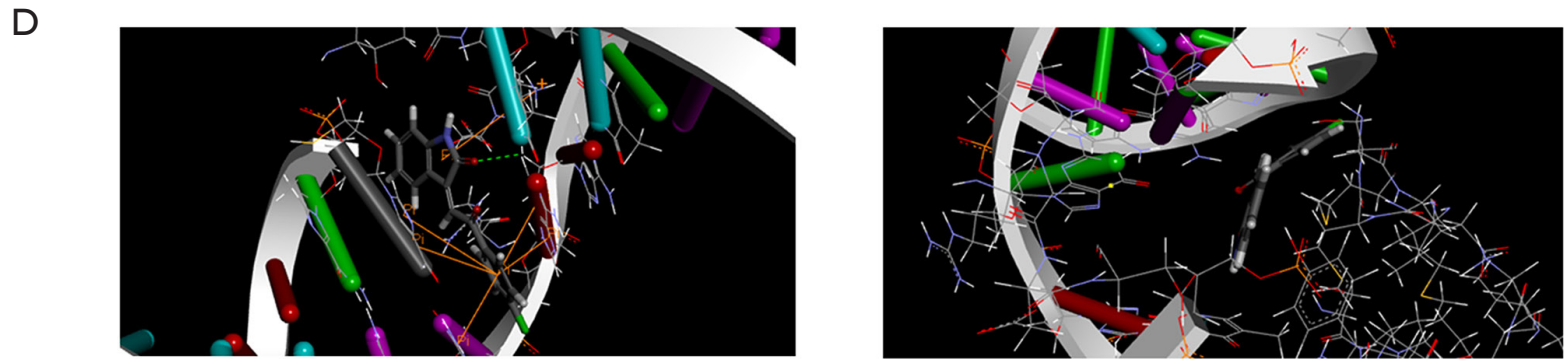

Figure 4 DIA-001 induces Top1 ccs accumulation. (A) Top1cc focus formation. The indicated U2OS cells treated with DIA-001 for 4 hours camptothecin for $0.5 \mathrm{~h}$ were stained with anti-Top1cc to detect Top1cc accumulation. DNA was co-stained with DAPI to visualize nuclei. (B,C) Effect of DIA-001 on DNA topoisomerase I and II. (B) Supercoiled pHOT-1 DNA (lane 1) and relaxed pHOT-1 DNA (lane 2) are both control experiments. Supercoiled pHOT-1 DNA without topoisomerase I (lane 3) incubation and Supercoiled pHOT-1 DNA was incubated with topoisomerase I combine $10 \mu \mathrm{M}$ (lane 5), $20 \mu \mathrm{M}$ (lane 6) DIA-001 and $50 \mu \mathrm{M}$ camptothecin (lane 4), respectively. (C) Linearized kDNA and decatenated kDNA are both control experiments. Catenated kDNA without topoisomerase (lane 3) II incubation and Catenated kDNA was incubated with topoisomerase II (lane 4) combine $10 \mu M$ (lane 6), $20 \mu M$ (lane 7) DIA-001 and $100 \mu M$ etoposide (lane 5), respectively. (D,E) Analysis the binding modes of DIA-001 in the topoisomerase I and II. Protein structure is shown in diagram.

topoisomerase I and topoisomerase II activities that participate in the winding or unwinding of DNA. Topo I inhibitors, such as CPT, are currently used in the treatment of cancer $(23,24)$ and always combine with radiotherapy that is standard treatment for cancer patients $(25,26)$. It has remarkable anticancer activity in the clinic, but also has some limitations with different cancer types. In vitro Topo I assay and molecular modelling study show the specific complex-based pharmacophores. The docked model of
DIA-001 with Top I-DNA complex, which is hydrogenbond formed between the ligand and residues are similar with CPT (23). The combination mode between DIA001 and Top I-DNA complex induced trapping of Top I by DIA-001 generous Top Icc. Finally, the two ends cannot be relegated induced DNA replication fork stop activated DNA damage pathway and apoptosis. Here we identified DIA-001, a novel Topo1 inhibitor, that is effective in the treatment of various cancers such as small cell lung 
carcinoma, oral squamous cell carcinoma and glioblastoma in vitro, but is comparatively not toxic for normal cells.

Based on the previous research, the anticancer effect of topoisomerase inhibitor is dependent on the pro-apoptotic and cell cycle arrest effect. Consistent with this, using U2OS cell line treated with DIA-001, we showed that PARP cleavage was enhanced. We also show that cyclin A levels increase after treatment with DIA-001 but cyclin B level increase only slightly suggesting cell cycle progression through the S/G2 transition but arrested at the G2/M phase (15-17). Together, the data from our study indicates that treatment with DIA-001 may induce cell cycle arrest in G2 phase and apoptosis in cancer cells.

In this study, we identified a new Topo1 inhibitor for cancer patient chemotherapy that induces DNA replication stress by accumulation of the Topo1cc on DNA and discuss the potential of using replication stress to promote the activation of DNA damage repair pathway, cell cycle arrest and apoptosis.

\section{Acknowledgments}

Funding: This work was supported by National Natural Science Foundation of China (91749115 to J Yuan, 81572770 to K Luo, 81972363 to S Zhao).

\section{Footnote}

Conflicts of Interest: The authors have no conflicts of interest to declare.

Ethical Statement: The authors are accountable for all aspects of the work in ensuring that questions related to the accuracy or integrity of any part of the work are appropriately investigated and resolved.

\section{References}

1. Nitiss JL. Investigating the biological functions of DNA topoisomerases in eukaryotic cells. Biochim Biophys Acta 1998;1400:63-81.

2. Wang JC. DNA topoisomerases. Annu Rev Biochem 1996;65:635-92.

3. Leppard JB, Champoux JJ. Human DNA topoisomerase I: relaxation, roles, and damage control. Chromosoma 2005;114:75-85.

4. Delgado JL, Hsieh CM, Chan NL, et al. Topoisomerases as anticancer targets. Biochem J 2018;475:373-98.
5. Pommier Y. DNA topoisomerase I inhibitors: chemistry, biology, and interfacial inhibition. Chem Rev 2009; 109:2894-902.

6. Xu Y, Her C. Inhibition of Topoisomerase (DNA) I (TOP1): DNA Damage Repair and Anticancer Therapy. Biomolecules 2015;5:1652-70.

7. Dusre L, Covey JM, Collins C, et al. DNA damage, cytotoxicity and free radical formation by mitomycin $\mathrm{C}$ in human cells. Chem Biol Interact 1989;71:63-78.

8. Bandi S, Viswanathan P, Gupta S. Evaluation of cytotoxicity and DNA damage response with analysis of intracellular ATM signaling pathways. Assay Drug Dev Technol 2014;12:272-81.

9. Bonner WM, Redon CE, Dickey JS, et al. GammaH2AX and cancer. Nat Rev Cancer 2008;8:957-67.

10. Tu WZ, Li B, Huang B, et al. $\gamma \mathrm{H} 2 \mathrm{AX}$ foci formation in the absence of DNA damage: mitotic $\mathrm{H} 2 \mathrm{AX}$ phosphorylation is mediated by the DNA-PKcs/CHK2 pathway. FEBS Lett 2013;587:3437-43.

11. Podhorecka M, Skladanowski A, Bozko P. H2AX Phosphorylation: Its Role in DNA Damage Response and Cancer Therapy. J Nucleic Acids 2010;2010. doi: 10.4061/2010/920161.

12. Chao HX, Poovey CE, Privette AA, et al. Orchestration of DNA Damage Checkpoint Dynamics across the Human Cell Cycle. Cell Syst 2017;5:445-59.e5.

13. Santivasi WL, Xia F. Ionizing radiation-induced DNA damage, response, and repair. Antioxid Redox Signal 2014;21:251-9.

14. Sakaue-Sawano A, Kobayashi T, Ohtawa K, et al. Druginduced cell cycle modulation leading to cell-cycle arrest, nuclear mis-segregation, or endoreplication. BMC Cell Biol 2011;12:2.

15. Shin DY, Sung Kang H, Kim GY, et al. Decitabine, a DNA methyltransferases inhibitor, induces cell cycle arrest at G2/M phase through p53-independent pathway in human cancer cells. Biomed Pharmacother 2013;67:305-11.

16. Hsiao YP, Tsai CH, Wu PP, et al. Cantharidin induces G2/ $M$ phase arrest by inhibition of Cdc25c and Cyclin A and triggers apoptosis through reactive oxygen species and the mitochondriadependent pathways of A375.S2 human melanoma cells. Int J Oncol 2014;45:2393-402.

17. Hiraoka D, Aono R, Hanada S, et al. Two new competing pathways establish the threshold for cyclin-B-Cdk1 activation at the meiotic G2/M transition. J Cell Sci 2016;129:3153-66.

18. McGowan AJ, Ruiz-Ruiz MC, Gorman AM, et al. Reactive 


\section{Page 8 of 8}

oxygen intermediate(s) (ROI): common mediator(s) of poly(ADP-ribose)polymerase (PARP) cleavage and apoptosis. FEBS Lett 1996;392:299-303.

19. Oliver FJ, de la Rubia G, Rolli V, et al. Importance of poly(ADP-ribose) polymerase and its cleavage in apoptosis. Lesson from an uncleavable mutant. J Biol Chem 1998;273:33533-9.

20. Aslan Koşar P, Tuncer H, Cihangir Uğuz A, et al. The efficiency of Poly(ADP-Ribose) Polymerase (PARP) cleavage on detection of apoptosis in an experimental model of testicular torsion. Int J Exp Pathol 2015;96:294-300.

21. Jain CK, Majumder HK, Roychoudhury S. Natural Compounds as Anticancer Agents Targeting DNA Topoisomerases. Curr Genomics 2017;18:75-92.

22. Pommier Y. Drugging topoisomerases: lessons and

Cite this article as: Liu J, Geng G, Liang G, Wang L, Luo K, Yuan J, Zhao S. A novel topoisomerase I inhibitor DIA-001 induces DNA damage mediated cell cycle arrest and apoptosis in cancer cell. Ann Transl Med 2020;8(4):89. doi: 10.21037/ atm.2019.12.138

\section{Liu et al. A novel top I inhibitor induces DNA damage in cancer cell}

challenges. ACS Chem Biol 2013;8:82-95.

23. Li F, Jiang T, Li Q, et al. Camptothecin (CPT) and its derivatives are known to target topoisomerase I (Top1) as their mechanism of action: did we miss something in CPT analogue molecular targets for treating human disease such as cancer? Am J Cancer Res 2017;7:2350-94.

24. Puddu F, Salguero I, Herzog M, et al. Chromatin determinants impart camptothecin sensitivity. EMBO Rep 2017;18:1000-12.

25. Yan Y, Li Z, Zeng S, et al. FGFR2-mediated phosphorylation of PTEN at tyrosine 240 contributes to the radioresistance of glioma. J Cell Commun Signal 2019;13:279-80.

26. Xu Z, Yan Y, Xiao L, et al. Radiosensitizing effect of diosmetin on radioresistant lung cancer cells via Akt signaling pathway. PLoS One 2017;12:e0175977. 